\title{
Zero-knowledge procedures for confidential access to medical records
}

\author{
Jean-Jacques Quisquater * André Bouckaert ** \\ *Philips Research Laboratory Belgium \\ Avenue Albert Einstein, 4 \\ B-1348 Louvain-la-Neuve, Belgium \\ jjq@prlb.philips.be \\ ** Université Catholique de Louvain \\ UMED - 72.25, Avenue E. Mounier \\ B-1200 Brussels, Belgium
}

\section{(Extended summary)}

During the seventies, several successful attempts were made to use computerized files for storing medical records, first by accessing to mainframes in large hospitals, and thereafter by use of microcomputers in decentralized medical services or general practitionners. The wide acceptance of computerized record keeping in general practice is witnessed e.g. by the fact there are more than fifty softwares available in the Belgian market for this purpose.

Such medical databases are vulnerable to spying: a hazard whose severity is increased by the traditional concept of medical secrecy (Mazen, 1988). According to this many-centuries-old concept, all medical data, or even non-medical data recorded during medical investigations, are not to be communicated by the doctor to anybody, except to another doctor if requested by the follow-up of the patient. Since paper medical records are very slowly and inefficiently retrieved, it could be thought that this ineffectiveness was a guarantee of confidentiality. This concept was not widely publicized but implicitly adopted and it explains the lack of security measures in most medical databases. There is no account of cases of piracy in medical databases although there is good reason to think that it has been increasingly practiced in the United States for litigation purposes.

Why not let the patients keep their own records? This question has been raised for the first time nine years ago (Metcalfe, 1980). Portable medical records were used episodicaly from the beginning of the eighties. Persons who advocated such 
portable records were usually public health doctors, motivated by the needs of the follow-up of the patients and, to a variable degree, by the feeling that it belongs to this patient to carry his own data or to be able to do so in a democratic society (Baldry \& al., 1986; Gentilini \& al., 1986; Bouckaert, 1984, 1988). The move towards portable records has been repeatedly criticized by medical professional unions and by their lobbies on the basis that such records would be vulnerable to blackmail.

In the European societies of the eighties, blackmailing a patient for his medical record is likely to be exercized by employers and by insurance companies. Other candidates to blackmail are police and security authorities, political parties, outlawed organizations. It should be understood that such a blackmail is always some kind of intermediate pressure, since the hard pressure is simply to ask for another medical examination. Nevertheless, it is not hard to imagine that an employed person could find it difficult to allow his employer to look at his record since the simple fact of refusing can be turned into a weapon against himself.

The concept of a portable computerized medical record stored on a smart card has evolved as a response to the dual challenge of confidentiality and portability. In the smart card, the access to the data is protected by access control rather than by enciphering although both methods can be used at the same time. In both cases, in order to access or/and to read, the usual approach is to request the production of a password by the patient. Such a patient password suffers from two weaknesses:

1. It exposes the patient to the hazard of blackmail.

2. It exposes the patient to the hazard of forgetting his own password.

At the same time, this policy achieves complete protection against the unauthorized use of stolen cards.

To avoid the blackmail, a possible solution is to use a two-key policy: both doctor and patient have a password and both use smart cards. Blackmail cannot be exercized against the patient without knowing the doctor's password. The main troubles are now:

1. The need for an external authority for password management, doctor habilitation, renewal procedures.

2. The possibility of monitoring the card readers to obtain the passwords.

3. The possibility of buffer spying.

4. The possibility of looking at the keyboard during password production. 
It is possible to avoid these dangers and to reduce the managerial burden of the external authority by using a zero-knowledge procedure (Fiat and Shamir, 1987; Guillou and Quisquater, 1988). With such a procedure, the doctor does not know his own password and does not need to produce it. The password of the doctor issued by an external authority that does not keep trace of its own production. Hence nobody but the doctor's smart card itself will know the password. The patient's smart card can nevertheless check that the doctor's smart card contains the valid secret information.

In this situation, the possibility of copying passwords disappears since the password is never actually revealed during the process of interaction between smart cards. But this policy gives no protection for stolen smart cards. If the doctor's password can only be checked by his own patients however, the possibility of stealing the doctor's and patient's card is quite reduced. If no restriction is made when the passwords are issued, the zero-knowledge procedure should be supplemented by enciphering and a password-conditional deciphering procedure or by an additional password-controlled access.

\section{References}

[1] M Baldry, G. Chéal, B. Fisher and al., Giving patients their own records in general practice: experience of patients and their staff, Brit. Med. J., 292, pp. 596-598, 1986.

[2] A. Bouckaert, Security of transportable computerized files, Advances in cryptology, Proceedings of EUROCRYPT '84, Springer-Verlag, pp. 416-425, 1985.

[3] A. Bouckaert, Medical records, confidentiality, smart cards, MIM News, 2, pp. 27-36, 1988 .

[4] J. L. Gentilini, J. L. Samaille, M. Trotin and P. Marquis, The PH-card: a portable and confidential medical file, Medinfo '86, Proceedings, pp. 10101014, Amsterdam 1986.

[5] N. J. Mazen, Le secret professionnel des praticiens de la santé, Vigot, Paris, 1988.

[6] D. H. Metcalfe, Why not let patients keep their own records?, J. Roy. Coll. Gen. Pract., 30, p. 420, 1980. 